\title{
Fragmentation in Molecular Clouds: The Formation of a Stellar Cluster
}

\author{
By RLF KLESSEN AND ANDREAS BURKERT
}

Max-Planck-Institut für Astronomie, Königstuhl 17, 69117 Heidelberg, Germany

\begin{abstract}
The isothermal gravitational collapse and fragmentation of a molecular cloud region and the subsequent formation of a protostellar cluster is investigated numerically. The clump mass spectrum which forms during the fragmentation phase can be well approximated by a power law distribution $d N / d M \propto M^{-1.5}$. In contrast, the mass spectrum of protostellar cores that form in the centers of Jeans unstable clumps and evolve through accretion and $N$-body interaction is best described by a log-normal distribution. Assuming a star formation efficiency of $\sim 10 \%$, it is in excellent agreement with the IMF of multiple stellar systems.
\end{abstract}

\section{Introduction}

Understanding the processes leading to the formation of stars is one of the fundamental challenges in astronomy and astrophysics. However, theoretical models considerably lag behind the recent observational progress. The analytical description of the star formation process is restricted to the collapse of isolated, idealized objects (Whitworth \& Summers 1985). Much the same applies to numerical studies (e.g. Boss 1997, Burkert et al. 1997 and reference therein). Previous numerical models that treated cloud fragmentation on scales larger than single, isolated clumps were strongly constrained by numerical resolution. Larson (1978), for example, used just 150 particles in an SPH-like simulation. Whitworth et al. (1995) were the first who addressed star formation in an entire cloud region using high-resolution numerical models. However, they studied a different problem: fragmentation and star formation in the shocked interface of colliding molecular clumps. While clump-clump interactions are expected to be abundant in molecular clouds, the rapid formation of a whole star cluster requires gravitational collapse on a size scale which contains many clumps and dense filaments.

Here, we present a high-resolution numerical model describing the dynamical evolution of an entire region embedded in the interior of a molecular cloud. We follow the fragmentation into dense protostellar cores which form a hierarchically structured cluster.

\section{Numerical Technique and Initial Condition}

To follow the time evolution of the system, we use smoothed particle hydrodynamics (SPH: for a review see Monaghan 1992) which is intrinsically Lagrangian and can resolve very high density contrasts. We adopt a standard description of artificial viscosity (Monaghan \& Gingold 1983) with the parameters $\alpha_{v}=1$ and $\beta_{v}=2$. The smoothing lengths are variable in space and time such that the number of neighbors for each particle remains at approximately fifty. The system is integrated in time using a second order Runge-Kutta-Fehlberg scheme, allowing individual timesteps for each particle. Once a highly condensed object has formed in the center of a collapsing cloud fragment and has passed beyond a certain density, we substitute it by a 'sink' particle which then continues to accrete material from its infalling gaseous envelope (Bate et al. 1995). By doing so we prevent the code time stepping from becoming prohibitively small. This procedure implies that we cannot describe the evolution of gas inside such a sink particle. For a 
detailed description of the physical processes inside a protostellar core, i.e. its further collapse and fragmentation, a new simulation just concentrating on this single object with the appropriate initial conditions taken from the larger scale simulation would be necessary (Burkert et al. 1998).

To achieve high computational speed, we have combined SPH with the special purpose hardware device GRAPE (Ebisuzaki et al. 1993), following the implementation described in detail by Steinmetz (1996). Since we wish to describe a region in the interior of a molecular cloud, we have to prevent global collapse. Therefore, we use periodic boundaries, applying the Ewald method in an PM-like scheme (Klessen 1997).

The structure of molecular clouds is very complex, consisting of a hierarchy of clumps and filaments on all scales (e.g. Blitz 1993). Many attempts have been made to identify the clump structure and derive its properties (Stutzki \& Güsten 1990, Williams et al. 1994). We choose as starting conditions Gaussian random density fluctuations with a power spectrum $P(k) \propto 1 / k^{N}$ and $0 \leq N \leq 3$. The fields are generated by applying the Zel'dovich (1970) approximation to an originally homogeneous gas distribution: we compute a hypothetical field of density fluctuations in Fourier space and solve Poisson's equation to obtain the corresponding self-consistent velocity field. These velocities are then used to advance the particles in one single big timestep $\delta t$. We present simulations with 50000 and $500000 \mathrm{SPH}$ particles, respectively.

\section{A Case Study}

As a case study, we present the time evolution of a region in the interior of a molecular cloud with $P(k) \propto 1 / k^{2}$ and containing a total mass of 222 Jeans masses determined from the temperature and mean density of the gas. Figure 1 depicts snapshots of the system initially, and when 10, 30 and 60 per cent of the gas mass has been accreted onto the protostellar cores. Note that the cube has to be seen periodically replicated in all directions. At the beginning, pressure smears out small scale features, whereas large scale fluctuations start to collapse into filaments and knots. After $t \approx 0.3$, the first highlycondensed cores form in the centers of the most massive and densest Jeans unstable gas clumps and are replaced by sink particles. Soon clumps of lower mass and density follow, altogether creating a hierarchically-structured cluster of accreting protostellar cores. For a realistic timing extimate, the Zel'dovich shift interval $\delta t=2.0$ has to be taken into account. In dimension-less time units, the free-fall time of the isolated cube is $\tau_{\mathrm{ff}}=1.4$.

\subsection{Scaling Properties}

The gas is isothermal. Hence, the calculations are scale free, depending only on one parameter: the dimensionless temperature $T \equiv E_{\text {int }} /\left|E_{\text {pot }}\right|$, which is defined as the ratio between the internal and gravitational energy of the gas. The model can thus be applied to star-forming regions with different physical properties. In the case of a dark cloud with mean density $n\left(H_{2}\right) \simeq 100 \mathrm{~cm}^{-3}$ and a temperature $T \simeq 10 \mathrm{~K}$ like Taurus-Auriga, the computation corresponds to a cube of length $10 \mathrm{pc}$ and a total mass of $6300 \mathrm{M}_{\odot}$. The dimensionless time unit corresponds to $2.2 \times 10^{6} \mathrm{yrs}$. For a high-mass star-forming region like Orion with $n\left(H_{2}\right) \simeq 10^{5} \mathrm{~cm}^{-3}$ and $T \simeq 30 \mathrm{~K}$ these values scale to $0.5 \mathrm{pc}$ and $1000 \mathrm{M}_{\odot}$, respectively. The time scale is $6.9 \times 10^{4} \mathrm{yrs}$.

\subsection{The Importance of Dynamical Interaction and Competitive Accretion}

The location and the time at which protostellar cores form, is determined by the dynamical evolution of their parental gas clouds. Besides collapsing individually, clumps stream towards a common center of attraction where they merge with each other or undergo 

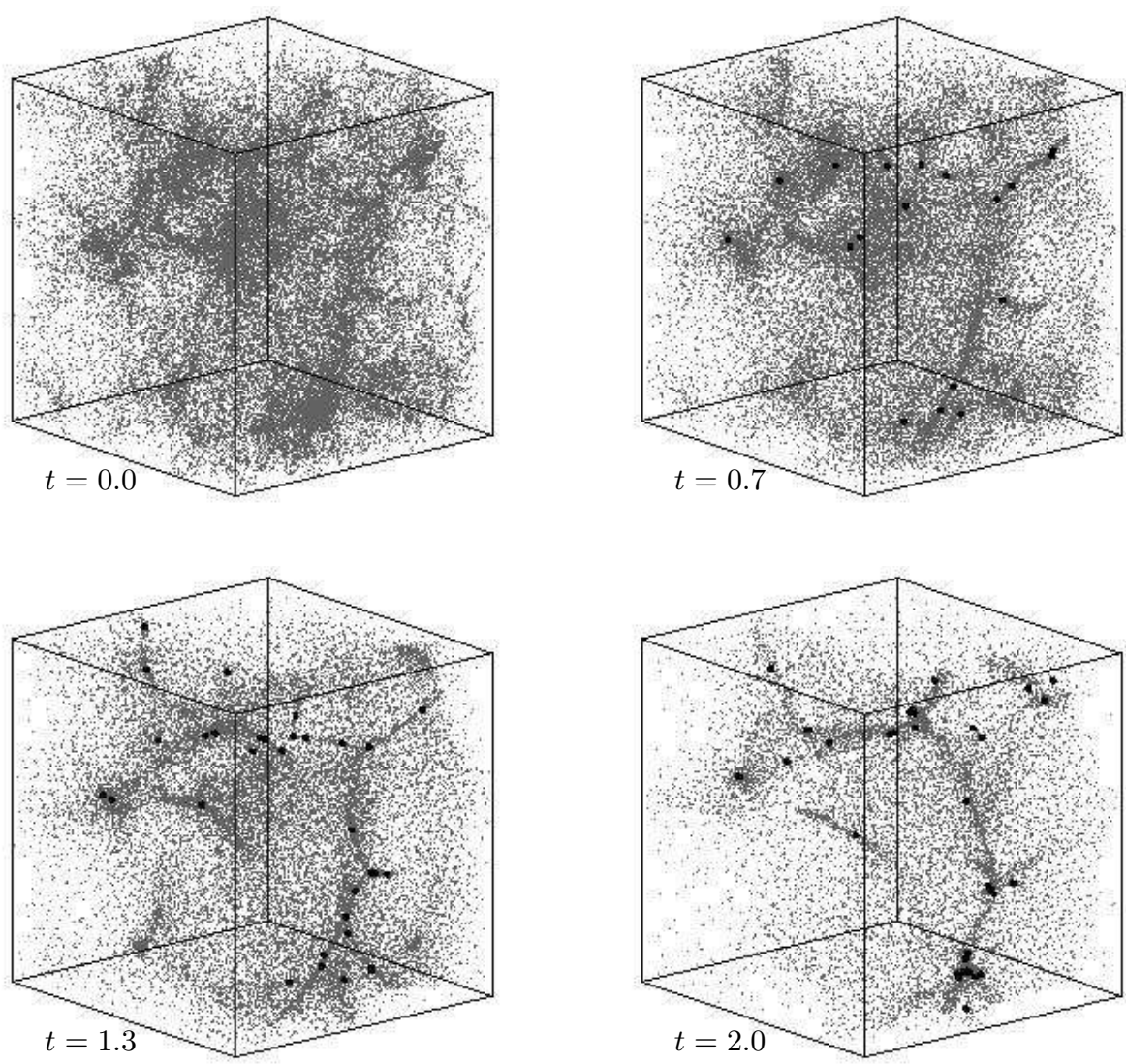

FiguRE 1. Time evolution and fragmentation of a region of 222 Jeans masses with initial Gaussian density fluctuations with power law $P(k) \propto 1 / k^{2}$. Collapse sets in and soon forms a cluster of highly-condensed cores, which continue to accrete from the surrounding gas reservoir. At $t=0.7$ about $10 \%$ of all the gas mass is converted into "protostellar" cores (denoted by black dots). At $t=1.3$ and $t=2.0$ these values are $30 \%$ and $60 \%$, respectively. Initially the cube contains $50000 \mathrm{SPH}$ particles.

further fragmentation. The formation of dense cores in the centers of clumps depends strongly on the relation between the timescales for individual collapse, merging and subfragmentation. Individual clumps may become Jeans unstable and start to collapse to form a condensed core in their centers. When clumps merge, the larger new clump continues to collapse, but contains now a multiple system of cores in its center. Now sharing a common environment, these cores compete for the limited reservoir of gas in their surrounding (see e.g. Price \& Podsiadlowski 1995, Bonnell et al. 1997). Furthermore, the protostellar cores interact gravitationally with each other. As in dense stellar clusters, close encounters lead to the formation of unstable triple or higher order systems and alter the orbital parameters of the cluster members. As a result, a considerable fraction of "protostellar" cores get expelled from their parental clump. Suddenly bereft of the massive gas inflow from their collapsing surrounding, they effectively stop accreting and their final mass is determined. Ejected objects can travel quite far and resemble the weak 

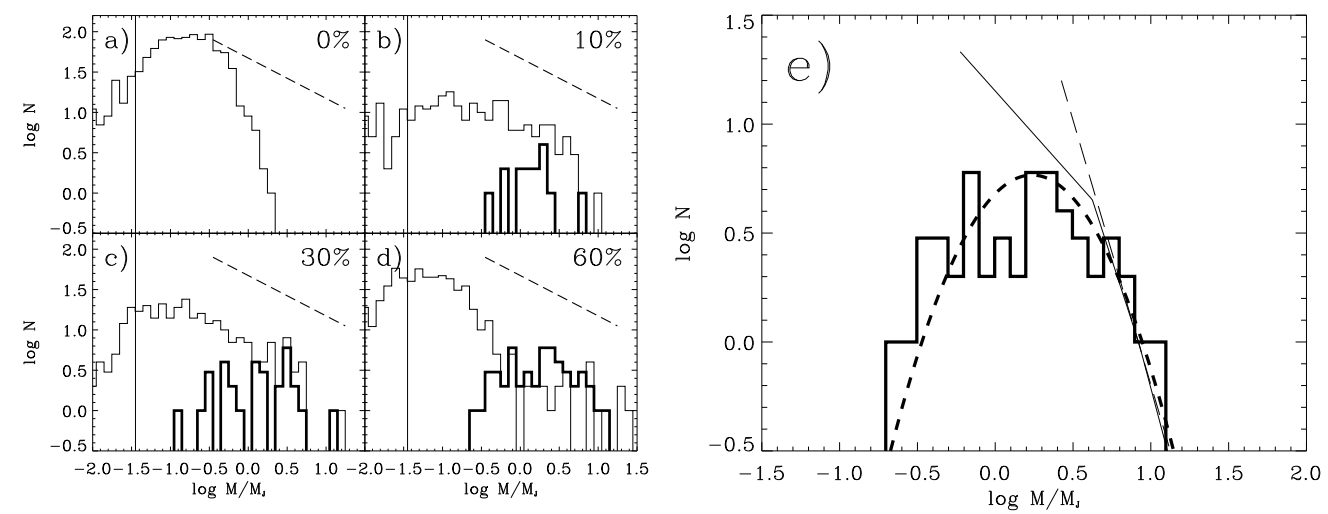

Figure 2. a) - d) Mass distribution of gas clumps (thin lines) and of protostellar cores (thick lines) at times $t=0.0,0.7,1.3$ and 2.0 when $0 \%, 10 \%, 30 \%$ and $60 \%$ of the total gas mass is condensed in cores, respectively. The vertical lines indicate the resolution limit of the simulation with 500000 particles (Bate \& Burkert 1997), and the dashed lines illustrate the observed clump mass spectrum with $d N / d M \propto M^{-1.5}$ (Blitz 1993). e) Comparison of the final core mass spectrum (thick line) with different observationally based models for the IMF. The thick dashed line denotes the log-normal form for the IMF, uncorrected for binary stars as proposed by Kroupa et al. (1990). In order for the peaks of both distributions to overlap, a core star formation efficiency of $10 \%$ has to be assumed. The agreement in width is remarkable. The multiple power-law IMF, corrected for binary stars (Kroupa et al. 1993) is shown by the thin solid line. As reference, the thin dashed line denotes the Salpeter (1955) IMF. Both are scaled to fit at the high-mass end of the spectrum. All masses are normalized to the overall Jeans mass in the system.

line T Tauri stars found via X-ray observation in the vicinities of star-forming molecular clouds (e.g. Neuhäuser et al. 1995).

\subsection{Mass Spectrum - Implications for the IMF}

Figures 2 $2 \mathrm{a}-\mathrm{d}$ describe the mass distribution of identified gas clumps (thin lines) and of protostellar cores (thick lines) that formed within unstable clumps in a simulation analogous to Fig. 1, but with 10 times higher resolution. To identify individual clumps we have developed an algorithm similar to the method described by Williams et al. (1994), but based on the framework of SPH. As reference, we also plot the observed canonical form for the clump mass spectrum, $d N / d M \propto M^{-1.5}$ (Blitz 1993), which has a slope of -0.5 when plotting $N$ versus $M$. Note that our initial condition does not exhibit a clear power law clump spectrum. The Zel'dovich approximation generates an overabundance of small scale fluctuations. However, in the subsequent evolution, these small clumps are immediately damped by pressure forces and non-linear gravitational collapse begins to create a power-law like mass spectrum.

A common feature in all our simulations is the broad mass spectrum of protostellar cores which peaks slightly above the overall Jeans mass of the system. This is somewhat surprising, given the fact that the evolution of individual cores is highly influenced by complex dynamical processes. In a statistical sense, the system retains 'knowledge' of its (initial) average properties.

The present simulations cannot resolve subfragmentation in condensed cores. Since detailed simulations show that perturbed cores tend to break up into multiple systems (e.g. Burkert et al. 1997), we can only determine the mass function of multiple systems. Our simulations predict an initial mass function with a log-normal functional form. Figure 2 2 e compares the results of our calculations with the observed IMF for mul- 
tiple systems from Kroupa et al. (1990). Assuming a typical Jeans mass $M_{\mathrm{J}} \approx 1 \mathrm{M}_{\odot}$ and a star formation efficiency of individual cores of $10 \%$, the agreement between the numerically-calculated mass function and the observed IMF for multiple systems (thick dashed line; from Kroupa et al. 1990) is excellent. For comparison, also the IMF corrected for binary stars (Kroupa et a. 1993) is indicated as thin solid line, together with the mass function from Salpeter (1955) as thin dashed line.

\section{Discussion}

Large-scale collapse and fragmentation in molecular clouds leads to a hierarchical cluster of condensed objects whose further dynamical evolution is extremely complex. The agreement between the numerically-calculated mass function and the observations strongly suggests that gravitational fragmentation and accretion processes dominate the origin of stellar masses. The final mass distribution of protostellar cores in isothermal models is a consequence of the chaotic kinematical evolution during the accretion phase. Our simulations give evidence, that the star formation process can best be understood in the frame work of a probabilistic theory. A sequence of statistical events may naturally lead to a log-normal IMF (see e.g. Zinnecker 1984, Adams \& Fatuzzo 1996; also Price \& Podsiadlowski 1995, Murray \& Lin 1996, Elmegreen 1997).

\section{REFERENCES}

Adams, R.C., Fatuzzo, M., 1995, ApJ, 464, 256

Bate, M.R., Burkert, A., 1997, MNRAS, 288, 1060

Bate, M.R., Bonnell, I.A, Price, N.M., 1995, MNRAS, 277, 362

Blitz, L., 1993, in Protostars and Planets III, eds. E.H. Levy \& J.J. Lunine, Univ. of Arizona

Bonnell, I.A., Bate, M.R., Clarke, C.J., Pringle, J.E., 1997, MNRAS, 285, 201

Boss, A., 1997, ApJ, 483, 309

Burkert, A., Bate, M.R., Bodenheimer, P., 1997, MNRAS, 289, 497

Burkert, A., Klessen, R.S., Bodenheimer, P., 1998, in The Orion Complex Revisited, eds. M. McCaughrean \& A. Burkert, ASP Conference Series, in press

Ebisuzaki, T., Makino, J., Fukushige, T., Taiji, M., Sugimoto, D., Ito, T., Okumura, S., 1993, PASJ, 45, 269

Elmegreen, B.G., 1997, ApJ, 486, 944

Klessen, R.S., 1997, MNRAS, 292, 11

Kroupa, P., Tout, C.A. \& Gilmore, G. 1990, MNRAS, 244, 76

Kroupa, P., Tout, C.A. \& Gilmore, G. 1993, MNRAS, 262, 545

Larson, R.B., 1978, MNRAS, 184, 69

Monaghan, J.J., 1992, ARAA, 30, 543

Monaghan, J.J., Gingold, R.A., 1983, J. Comp. Phys.,52, 135

Murray, S.D., Lin, D.N.C., 1996, ApJ, 467, 728

Neuhäuser, R., Sterzik, M.F., Schmitt, J.H.M.M., Wichmann, R., Krautter, J., 1995, A\&̋A, 297, 391

Price, N.M., Podsiadlowski, Ph., 1995, MNRAS, 273, 1041

Salpeter, E.E. 1955, ApJ, 121, 161

Steinmetz, M., 1996, MNRAS, 278, 1005

Stutzki, J., Güsten, R., 1990, ApJ, 356, 513

Williams, J.P, De Geus, E.J., Blitz, L., 1994, ApJ, 428, 693

Whitworth, A.P., Summers, D., 1985, MNRAS, 214, 1

Whitworth, A.P., Chapman, S.J., Bhattal, A.S., Disney, M.J., Pongracic, H., Turner, J.A., 1995, $M N R A S, \mathbf{2 7 7}, 727$

Zel'dovich, Y.B., 1970, $A \& A, \mathbf{5}, 84$

Zinnecker, H., 1984, MNRAS, 210, 43 